\title{
Study on the Sport Training Supplementary System Based on DSS
}

\author{
Qiang LI \\ Department of Physical education, North-west University, Xi'an, 710069, China \\ 23414388@qq.com
}

Keywords: DSS, Sport Training, Supplementary System.

\begin{abstract}
According to the need or modern sport training, we developed a framework of sport training supplementary system based on DDS techniques and relevant ideas of software engineering, and analyzed the component and functions. We aim at satisfying the need of sport training and the daily processing of data for management by the decision mode combining qualltative and quantitative methods, in order to improve the scientific degree of sport training.
\end{abstract}

\section{Introduction}

Contemporary theory and technology of high-tech sports training field has comprehensively become the main tendency of the sport development. Advanced scientific theories can help the development of athletes more systemic, scientific and money-saving, which deeply dig the best match of athletes' potentials in every aspect and improve their sport scores greatly. Decision support system (DDS) is a new application subject to computer science, with the development of software engineering. The main characteristic of DDS is the solution of the half structural problems. It can provide the supplemental function via the connection of human-computer. Its target is to help people resolve the decision process and improve the effectiveness of organizations. The function of the theory is to make up relevant data that is related to solving the decision problems. And the second function is to collect, store and provide the data outside the system in a real time. Thirdly, it can store and study the models for decisions in a certain way of storing and saving[1]. In sum, it helps people to make decision and improve the decision techniques and levels, which decides that DDS has a wide potential applications as an advanced tool for sport training. Here we use DDS techniques to sustain the decision support system, in order to make the sport training more scientific, systemic and intelligent. We aim at providing a powerful decision tool that can highly improve the scientific level of sport training.

\section{Organization of the Text}

\section{Basic Pattern of Decision Support System}

The basic pattern of a complete decision support system contains the decision support system, real system, outside environment and the decision maker. The decision maker is the core and they can use their knowledge and expertise to output the response of the decision support system. And also, the decision maker can analyze the real system and make decisions[2]. During the decision making process, assistants can provide help to decision makers. To the real system, the data of questions and operations is the output information flow, and the decision of the decision maker is the input information flow[3].

In Figure 1, the basement of the figure shows the basic data of the decision support system, including the processed information from the real system, information of environment, information related to people's behaviors and the statistical information. The right of the figure is the decision support system, including the database system, model base system and the man-machine interactive system. Decision maker can use their own knowledge and expertise to make decision for the real system combining the output of the decision support system. 


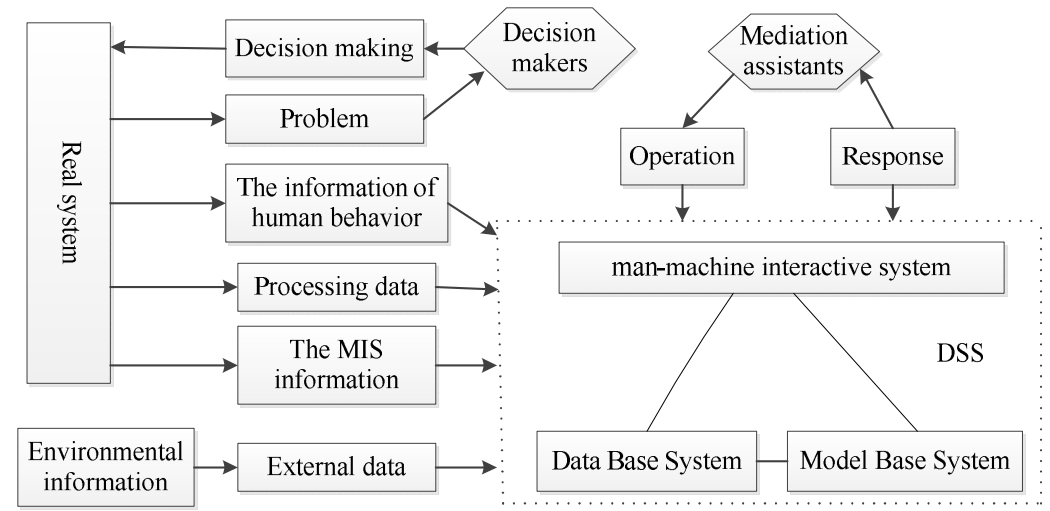

Fig. 1 The basic model of decision support system

\section{Development of the Sport Training Supplementary System Based on DSS}

Overall Target. Sports training aimed at athletes by changing the functional state of the body, so as to achieve adaptation and external behavior at a higher level. When the physical condition of athletes generate new adaptation, the level of exercise training will enter a higher level. If you neglect to adapt sports training and functional state of the body, exercise training cannot reach the best results. Therefore, a sufficient evaluation to the athletes' statuses provided by the system can help coaches comprehensively, precisely and quickly grasp the athletes' situations and then make reasonable arrangements and adjustments to the trainings, which can help athletes acquire the best status for sports. Here, our studied system can follow the simple, effective and operable principles according to the actual sport training, to make decision based on the observation of athletes' training status and relevant indicators. Finally, it provides reasonable suggestions and training plans. Figure 2 is the framework of the sport training supplementary system.

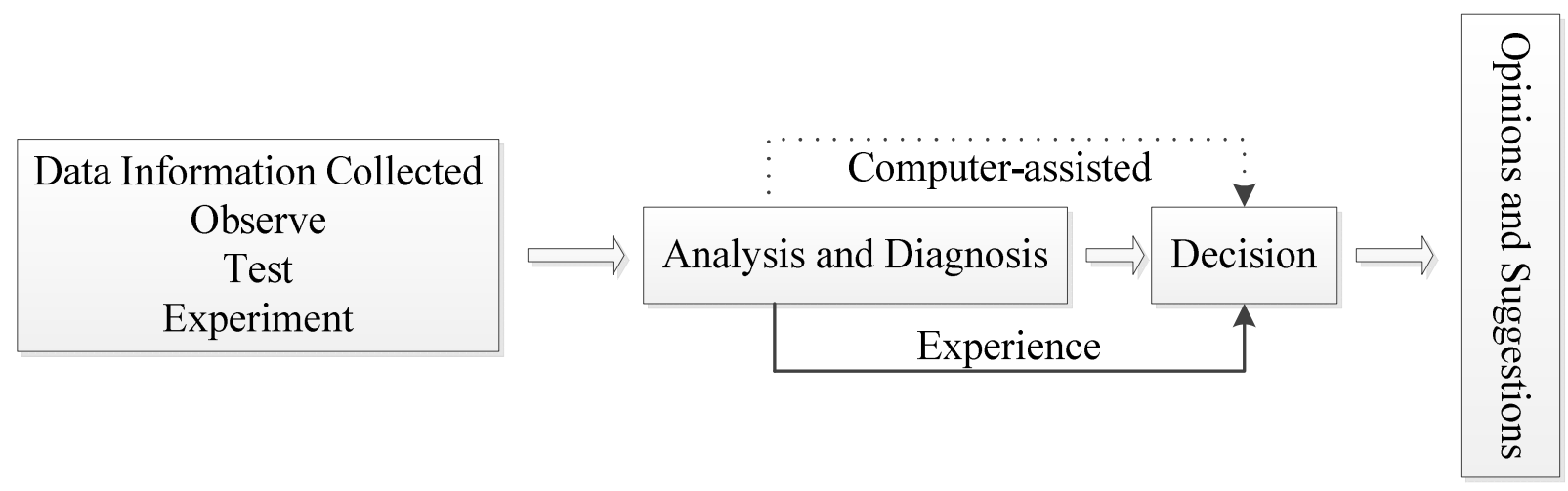

Fig. 2 The framework of the sport training supplementary system

Structure of Content. Here we develop the sport training supplementary system combining the need of practical training needs and the consideration of the content structure of the system, including the comprehensive management of athletes, the supervision of training loads, evaluation of body status, self-supervision, nutrition and hurts. As shown in figure 3. 


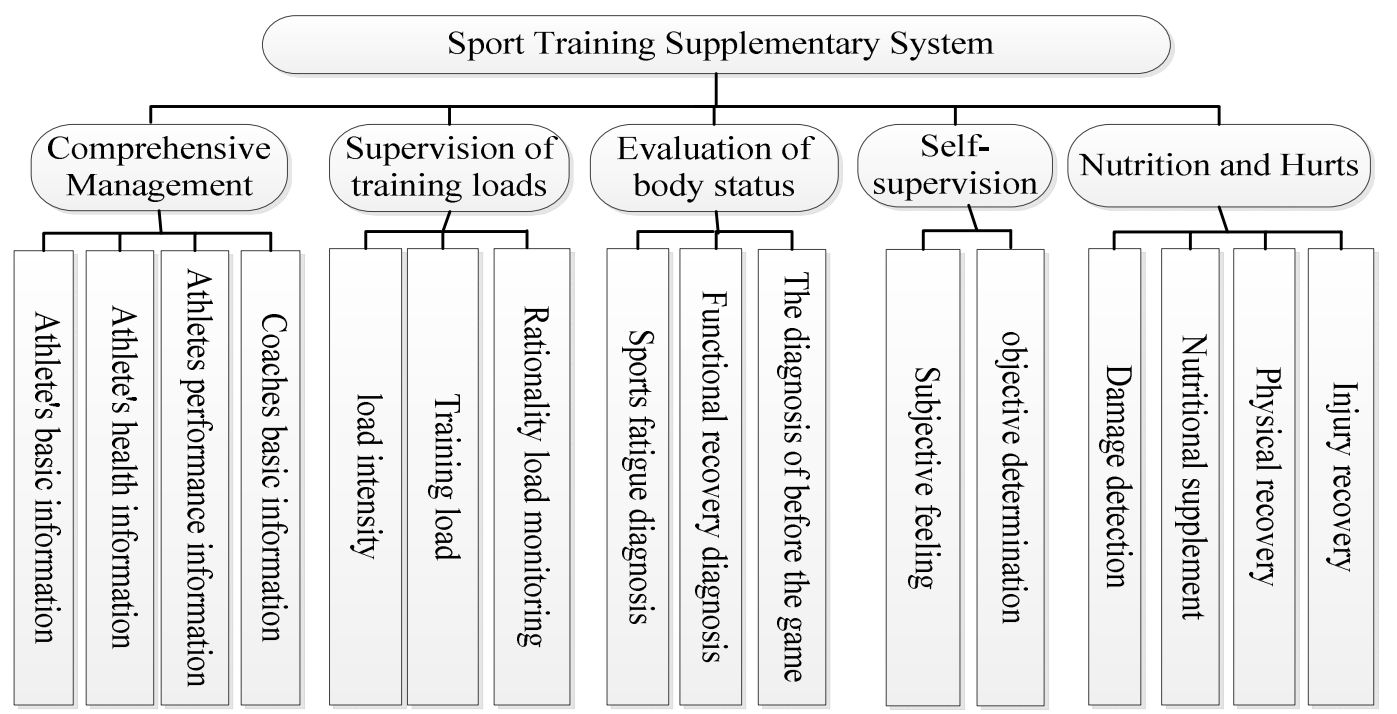

Fig. 3 The general structure of auxiliary system in sports training

Design of System. Decision support system for the design phase of the system must first determine the design of specialized physical components, structure and development platform, then the whole system design process is divided into preliminary design and detailed design[4]. Decision Support System is the completion of the preliminary design of the overall system design, for problem decomposition and synthesis problems. The detailed design of decision support system mainly on data and models for detailed design, design data, including data files and database designs. Model design includes model algorithm design and model library design.

The study of decision support system is designed primarily to system data, models and knowledge. During the data design, the design is in the form of the design of database. Model design includes the designs of the data instruction files and the model instruction files. Mathematical models are in the form of mathematical equations and the equations are put forward with the design of algorithms. Programming is designed after the effective algorithm designed of the model. Design of sports training decision support system for monitoring function in the program uses the database, model base, knowledge base to cooperate more technology, through a unified interface management, realizing the functions of sports training function diagnosis and evaluation, training, control system calls. The summary case of the structure is shown as Figure 4.

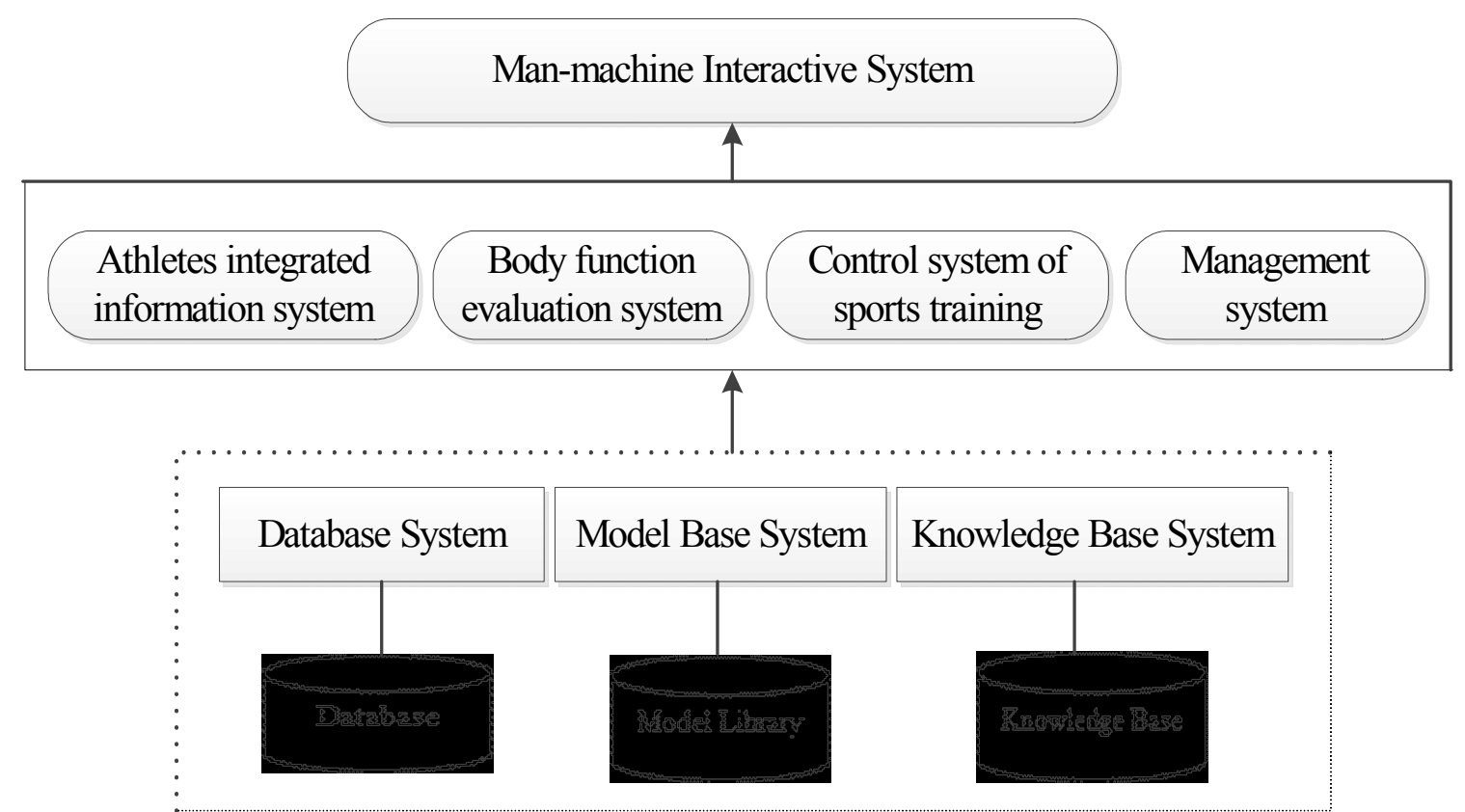

Fig. 4 The DSS structure of sport training supplementary system 
Design of Man-Machine Interactive System. Man-machine interactive system is the man-machine interface, human-computer interface and user-machine interactive component, which is a core part of a decision support system, connecting the man and the system. The system is linked to the user database, model base and knowledge base, providing interactive forms of decision-making to decision makers in various forms[5]. The initial interface of the system is composed of four sub-systems modules, including athletes comprehensive information management system, Athletes' physical evaluation system, sports training control systems and management systems. Processing system with its interface to interact with the computer language of action by the user interface management system handles the user interface management system and allows users to interact with the model and database subsystems, and also, operate results from the display language to the user. The used user interface mode includes interactive menus, command language, questions and answers, interactive forms, natural language processing, graphical user interfaces, and mixed mode.

Design of Database. The database is an important part of decision support systems. Decision support systems have special requirements to the database. These requirements mainly include the following aspects: ability to extract the data, the level of detail to support the data changes, support for memory, multiple data sources, set operations, and support a variety of relationships and view other parts of the decision support system has a good interface, and end-users have a good connection. Therefore, to achieve these DSS database requirements, the database from a functional perspective should includes the following five components: data extraction subsystem, DSS database, database management systems, data dictionary and data query module.

Design of Model Library. Model Library plays an important role in decision support systems. DSS is not directly dependent on the use of data in the database to make decisions, but on the model library model decision-making. The ability to model base for decision-making analysis is the inference, comparison and selection decision analysis capabilities to answer questions. Model library system includes model libraries, model management systems and models dictionaries, the main function is to let decision makers be in a variety of models using the model library to support decision making.

In the model library system, we must first consider the model representation and store it in a computer form, which makes the model easy to manage, high efficiency, and can be flexibly combined. In order to enhance management flexibility and reduce storage redundancy representation, the model tends to break down into basic elements of the model, a combination of a basic unit model. Therefore, the establishment of sports training DSS various models, such as the training load model, the functional state of the diagnostic model, athletic performance prediction models, we should first select it in the form, and then determine the necessary format description model range, including the number of variables, meaning, location, and other operations, and the number and meaning of the various parameters as well as coefficients.

Design of Knowledge Base System. Knowledge base is a key component to achieve intelligent decision support system. The design of knowledge base is the advanced stage of the development of the design of the DDS. In general, the design work should build the knowledge base on the development of decision support systems, the latter stage includes the preparation, system analysis, prototyping, improvement, expansion and maintenance.

To facilitate the use and maintenance, sports training aids system encompasses many aspects of knowledge. The knowledge base can be divided into the decision base and the information base. The decision base stores the rule type knowledge and rules based knowledge. The used mechanism can grow gradually during the utilization. Information base stores the factual knowledge, including common movement disorders, commonly used indicators of exercise training, proper nutrition athletes, athletes weight loss, regeneration guidance and the development of different specialized training methods. Functional information management module is to provide decision support to sports training function monitoring system, which consists of three modules: knowledge inquiry, publish knowledge of information, knowledge and information management information. Decision 
makers can access to competitive sports, sports training, fitness and other related knowledge according to their knowledge and information that will be released to the decision support system.

\section{Summary}

The computer-aided sports training system can deeply combine the physical training and computer applications. Using computer acquisition, storage and analysis of training data, the system can help coaches manage daily training information, training aid decisions, which is an important part of the movement to achieve full control of the training process. Computer-aided exercise training through interdisciplinary research of science, sports medicine, biomechanics and information science and other disciplines of sports training, can improve training efficiency and level of training, avoiding the traditional reliance of training deficiencies. It provides a unified platform of data analysis for decision coaches, researchers and other decision-makers at different levels, offering a scientific basis to quantify training load, training methods, training objectives and various optimization program. Hence it can support the decisions of coaches and researchers with a more scientific way.

\section{References}

[1] H. Li, W. Leng, Y. Zhou, F. Chen, Z. Xiu, D. Yang, Evaluation models for soil nutrient based on support vector machine and artificial neural networks, The Scientific World Journal, 2014 (2014) 478569.

[2] Y Q. Dong, W. Guo, An overview of the DSS system, Network \& Information, 6(2007)78-79.

[3] Y. Tan, Decision support system, first ed., Electronic Industry Press, Bei Jing, 2011.

[4] Sébastien, Guillet,Bruno, Correct by Construction Security Approach to Design Fault Tolerant Smart Homes for Disabled People, Procedia Computer Science, 17(2013 )123-126.

[5] H. Li, X. Wang, T. Yi, Z. Xu, X. Liu, Prediction of Henry's law constants for organic compounds using multilayer feedforward neural networks based on linear solvation energy relationship, Journal of Chemical \& Pharmaceutical Research, 6 (2014) 1557-1564. 